\title{
ABELIAN SUBGROUP SEPARABILITY OF SOME ONE-RELATOR GROUPS
}

\author{
D. TIEUDJO
}

Received 29 August 2004 and in revised form 11 June 2005

We prove that any group in the class of one-relator groups given by the presentation $\left\langle a, b ;\left[a^{m}, b^{n}\right]=1\right\rangle$, where $m$ and $n$ are integers greater than 1 , is cyclic subgroup separable (or $\pi_{c}$ ). We establish some suitable properties of these groups which enable us to prove that every finitely generated abelian subgroup of any of such groups is finitely separable.

\section{Introduction}

Following Malcev [5], we will call a subgroup $M$ of a group G finitely separable if for any element $g \in G$, not belonging to $M$, there exists a homomorphism $\varphi$ of group $G$ onto some finite group $X$ such that $g \varphi \notin M \varphi$. This is equivalent to the statement that for any element $g \in G \backslash M$, there exists a normal subgroup $N$ of finite index in $G$ such that $g \notin M N$.

A group $G$ is subgroup separable if each of its finitely generated subgroups is finitely separable. If every cyclic (or finitely generated abelian) subgroup of a group $G$ is finitely separable, then group $G$ is said to be cyclic (resp., abelian) subgroup separable. Cyclic subgroup separable groups are also called $\pi_{c}$ groups.

Evidently, every $\pi_{c}$ group is residually finite (i.e., any of its one-element subsets is finitely separable). However, the converse is not true. So, cyclic subgroup separability strengthens residual finiteness. But subgroup separability is stronger; in fact, if $G$ is a finitely presented subgroup separable group, then $G$ has a solvable generalized word problem (just as if $G$ is a finitely presented residually finite group, then $G$ has a solvable word problem). So subgroup separability is so strong that very few classes of groups satisfy it. But cyclic subgroup separability is possessed by much larger classes of groups.

Hall in [1] showed that free groups are $\pi_{c}$. In [5], Malcev proved that finitely generated torsion-free nilpotent groups are $\pi_{c}$. Free products of $\pi_{c}$ groups are $\pi_{c}$; further, it was established that finite extensions of $\pi_{c}$ groups are $\pi_{c}[6,7]$. Cyclic subgroup separability of certain generalized free products of $\pi_{c}$ groups amalgamating a cyclic subgroup was proved in $[10,8]$. Some one-relator groups happen to be $\pi_{c}[10,11]$.

In this paper, we enlarge the classes of one-relator $\pi_{c}$ groups. We will prove the following theorem. 
Theorem 1.1. Any group $G_{m n}=\left\langle a, b ;\left[a^{m}, b^{n}\right]=1\right\rangle$, where $m$ and $n$ are integers greater than 1 , is $\pi_{c}$.

As we will see, any of groups $G_{m n}$ is a special type of generalized free product of $\pi_{c}$ groups amalgamating a finitely generated abelian subgroup. We will establish the following property of these groups.

THeORem 1.2. Every abelian subgroup of any of groups $G_{m n}(m, n>1)$, which contains a cyclically reduced element of length greater than 1 , is cyclic.

Therefore, the next corollary, which is the aim of this paper, is obvious.

COROLLARY 1.3. Every finitely generated abelian subgroup of $G_{m n}$, where $m$ and $n$ are integers greater than 1, is finitely separable.

We note that the assertion of the corollary is also valid when $m=1$ or $n=1$. Indeed, we can use the results in [10] or [11] to see it.

Our interest in the class of groups $G_{m n}$ is that these groups are "very well behaved." Their residual finiteness is well known; it follows, for example, from the results of [2]. Thus, groups $G_{m n}$ are Hopfian (i.e., each of their surjective endomorphisms is an automorphism) since they are finitely generated and residually finite [4]. Some properties of these groups were considered in [9] where, in particular, the description of their endomorphisms was given.

The proofs in $[2,9]$ made use of the presentation of group $G_{m n}$ as amalgamated free product. The same presentation will be the crucial tool in the proof of the results stated above.

\section{Preliminaries on amalgamated free products}

We recall some notions and properties concerned with the construction of free product $P=(X * Y ; U)$ of groups $X$ and $Y$ amalgamating subgroup $U$.

Every element $g \in P$ can be written in the following form:

(I)

$$
g=x_{1} x_{2} \cdots x_{r} \quad(r \geqslant 1)
$$

where for any $i=1,2, \ldots, r$, element $x_{i}$ belongs to one of the free factors $X$ or $Y$ and if $r>1$, any consecutive $x_{i}$ and $x_{i+1}$ do not belong to the same factor $X$ or $Y$ (nor the amalgamated subgroup $U)$. Any such form of element $g$ is called reduced.

In general, an element of group $P=(X * Y ; U)$ can have more than one reduced form. Further if $g=y_{1} y_{2} \cdots y_{s}$ is one more reduced form of $g$, then $r=s$ and, for any $i=$ $1,2, \ldots, r, x_{i}$ and $y_{i}$ belong to the same factor $X$ or $Y$; more precisely, there exist elements $u_{0}, u_{1}, \ldots, u_{r}$ of $U$ such that

(II)

$$
x_{i}=u_{i-1}^{-1} y_{i} u_{i} \quad(1 \leq i \leq r) u_{0}=u_{r}=1 .
$$

Thus, any two reduced forms of an element $g$ of a group $P=(X * Y ; U)$ have the same number of components, which we will call the length of element $g$ and denote by $l(g)$. 
Remark 2.1. The length of an element introduced here is different from that in [3]. Here, elements of length 1 are elements of the free factors, whereas elements of the amalgamated subgroup have length 0 according to [3].

An element $g$ is called cyclically reduced if either $l(g)=1$ or $l(g)>1$ and the first and the last components of its reduced form do not belong to the same factor $X$ or $Y$.

In the group $P$, any element $g$ which is not cyclically reduced can be written as

$$
g=u \cdot v \cdot u^{-1}
$$

where $u$ and $v$ are elements of $P$ with reduced forms $u=x_{1} x_{2} \cdots x_{r}$ and $v=y_{1} y_{2} \cdots y_{s}$, and element $v$ is cyclically reduced. In these forms, elements $x_{r}$ and $y_{1}$ do not belong to the same subgroup $X$ or $Y$ and if $s>1$, element $y_{s} x_{r}^{-1}$, which lies in the same subgroup $X$ or $Y$ as elements $x_{r}$ and $y_{s}$, does not belong to subgroup $U$.

Since a given group $G$ is $\pi_{c}$ if and only if for every pair of its elements $f$ and $g$ either $f=$ $g^{k}$ for some integer $k$ or there exists a normal subgroup $N$ of finite index in $G$ such that $f \not \equiv g^{t}(\bmod N)$ for all integers $t$, we state the following tool, analogous to [10, Lemma $1]$.

Proposition 2.2. Let $P=(X * Y ; U)$ be the free product of groups $X$ and $Y$ amalgamating subgroup $U$. Let $f, g \in P$ such that $f=x_{1} x_{2} \cdots x_{r}$ and $g=y_{1} y_{2} \cdots y_{s}$. If $f$ is reduced and $g$ is cyclically reduced, then $f \in\langle g\rangle$ only under the following conditions.

(1) Elements $f$ and $g$ belong to the same free factor $X$ or $Y$ and the equality $f=g^{k}$ is satisfied in that free factor, for some integer $k \neq 0$.

(2) Elements $f$ and $g$ belong to different subgroups $X$ and $Y$ and the equality $f=g^{k}$ is satisfied in the amalgamated subgroup $U$, for some integer $k \neq 0$.

(3) $l(f), l(g)>1$ and $l(g)$ divides $l(f)$; in this case, if $f=g^{k}$ for some positive integer $k \neq 0$, then $r=k$ s and there exist elements $u_{0}, u_{1}, \ldots, u_{k s}$ of subgroup $U$ such that

(P)

$$
\begin{gathered}
x_{i}=u_{i-1}^{-1} y_{i} u_{i}, \quad \text { for } i=1,2, \ldots, k s, \text { where } \\
y_{j}=y_{j+l s} \quad \text { for } j=1,2, \ldots, s, 0 \leq l \leq k-1, u_{0}=u_{k s}=1 .
\end{gathered}
$$

If integer $k$ is negative, then similar equations hold when replacing elements $y_{k s}^{-1}$ by $y_{1}, y_{k s-1}^{-1}$ by $y_{2}$, and so on.

Proof. Let $f=x_{1} x_{2} \cdots x_{r}$ be reduced and let $g=y_{1} y_{2} \cdots y_{s}$ be cyclically reduced. If $s=$ $l(g)>1$, then for any integer $n \neq 0, l\left(g^{n}\right)=|n| \cdot l(g)$. Therefore, if $f=g^{k}$ for some integer $k \neq 0$, then either $l(f)=l(g)=1$ or $l(f)>1$ and $l(g)>1$.

Consequently, we have the following possibilities.

Case 1. $l(f)=l(g)=1$.

Subcase $i$. Elements $f$ and $g$ can belong to the same free factor $X$ or $Y$, and hence the equality $f=g^{k}$ takes place in this factor. This corresponds to case (2) in the proposition. Subcase ii. Let elements $f$ and $g$ belong to different factors. For example, let $f \in X$ and $g \in Y$; then $g^{k} \in Y$ and $X \ni f=g^{k} \in Y$, thus $f=g^{k} \in X \cap Y=U$. The equality $f=g^{k}$ 
now holds in the amalgamated subgroup $U$ of the group $P$. Hence, (2) in the formulation of the proposition is satisfied.

Case 2. $l(f)>1$ and $l(g)>1$.

Since $g$ is cyclically reduced, $g^{k}$ is cyclically reduced and $l\left(g^{k}\right)=k s$. So, the equality $f=g^{k}$ implies that $r=k s$ and

$$
x_{1} x_{2} \cdots x_{r}=y_{1} y_{2} \cdots y_{s} y_{1} y_{2} \cdots y_{s} \cdots y_{1} y_{2} \cdots y_{s}
$$

which we can write as

(*)

$$
x_{1} x_{2} \cdots x_{r}=y_{1} y_{2} \cdots y_{s} y_{s+1} y_{s+2} \cdots y_{k s}
$$

where $y_{j}=y_{j+l s}$ for $j=1, \ldots, s$ and $0 \leq l \leq k-1$. So, by applying conditions of equalities (2.2), there exists a sequence of elements $u_{0}, \ldots, u_{k s}$ of subgroup $U$ such that

$$
x_{i}=u_{i-1}^{-1} y_{i} u_{i}, \quad \text { for } i=1, \ldots, k s, \quad u_{0}=u_{k s}=1 .
$$

Thus, $x_{i}=u_{i-1}^{-1} y_{i} u_{i}$ for $i=1,2, \ldots, k s$, where $y_{j}=y_{j+l s}$, for $j=1,2, \ldots, s$ and $0 \leq l \leq k-1$, and $u_{0}=u_{k s}=1$. So, case (3) in the formulation of the proposition is satisfied.

If integer $k$ is negative, then replacing in (2.6) elements $y_{k s}^{-1}$ by $y_{1}, y_{k s-1}^{-1}$ by $y_{2}$, and so on, we obtain equations similar to (2.4). Hence, the proposition is proven.

\section{Structure and some subgroups and quotients of groups $G_{m n}$}

We now establish some preliminary properties of groups of kind

$$
G_{m n}=\left\langle a, b ;\left[a^{m}, b^{n}\right]=1\right\rangle
$$

where $m>1$ and $n>1$. We begin by describing the construction of such groups as amalgamated free products.

Let

$$
H=\langle c, d ; c d=d c\rangle
$$

be the free abelian group of a rank 2 . We denote by $\langle x\rangle$ the infinite cycle, generated by element $x$.

We denote by $A$ the free product of groups $\langle a\rangle$ and $H$, amalgamating subgroups $\left\langle a^{m}\right\rangle$ and $\langle c\rangle$, through the isomorphism carrying element $a^{m}$ into element $c$. Thus, group $A$ has the presentation

$$
A=\left\langle a, c, d ; c d=d c, a^{m}=c\right\rangle .
$$


Similarly, we define the group

$$
B=\left\langle b, c, d ; c d=d c, b^{n}=d\right\rangle,
$$

which is the free product of groups $\langle b\rangle$ and $H$, amalgamating subgroups $\left\langle b^{n}\right\rangle$ and $\langle d\rangle$.

By using Tietze transformations, one proves that the free product of groups $A$ and $B$, amalgamating subgroup $H$, coincides with group $G_{m n}$. Thus,

$$
G_{m n}=(A * B ; H),
$$

where

$$
A=\left(\langle a\rangle * H ; a^{m}=c\right), \quad B=\left(\langle b\rangle * H ; b^{n}=d\right)
$$

These notations are fixed everywhere below. So the length of an element should be considered in the decomposition of the indicated group as free product with amalgamated subgroups.

From the decomposition of groups $A, B$ and $G_{m n}$ as free product with amalgamated subgroup and from the properties of this construction, it follows that these groups are torsion-free. Further, [3, Corollary 4.5] permits to prove, that the center of group $A$ coincides with subgroup $\langle c\rangle$, the center of group $B$ is subgroup $\langle d\rangle$, and the center of group $G_{m n}$ is trivial.

Since the amalgamated subgroups in the decomposition of groups $A$ and $B$ are central in the free factors, it can be checked immediately that the following proposition holds.

Proposition 3.1. For any element $g$ of group A, not belonging to subgroup $\mathrm{H}, \mathrm{g}^{-1} \mathrm{Hg} \cap$ $H=\langle c\rangle$. Similarly, $g^{-1} H g \cap H=\langle d\rangle$ for $g \in B \backslash H$. In particular, for any element $g$ of $A$ or $B$ and any element $h \in H, g^{-1} h g \in H$ if and only if $g$ and $h$ commute.

Our next goal is to build some subgroups and quotients of group $G_{m n}$ and examine some of their properties.

Let $t$ be an integer greater than 1 . We denote by $H^{t}$ the subgroup of $H$, consisting of elements $h^{t}$, where $h \in H$. It is easy to see that the factor group $H / H^{t}$, denoted by $H(t)$, is the direct product of two cyclic subgroups of order $t$, generated by elements $c H^{t}$ and $d H^{t}$, respectively. Since the order of $a^{m}$ in the group $\left\langle a ; a^{m t}=1\right\rangle$ is also equal to $t$, we can construct the free product

$$
A(t)=\left(\left\langle a ; a^{m t}=1\right\rangle * H(t) ; a^{m}=c H^{t}\right)
$$

of groups $\left\langle a ; a^{m t}=1\right\rangle$ and $H / H^{t}$ amalgamating subgroup $\left\langle a^{m}=c H^{t}\right\rangle$. Since the canonical homomorphisms of the group $\langle a\rangle$ onto the group $\left\langle a ; a^{m t}=1\right\rangle$ and of $H$ onto the factor group $H / H^{t}$ coincide on the amalgamated subgroup, (relative to the decomposition of group $A$ as amalgamated free product), there exists a homomorphism $\rho_{t}$ of group $A$ onto group $A(t)$, extending the indicated canonical mappings.

Similarly,

$$
B(t)=\left(\left\langle b ; b^{n t}=1\right\rangle * H(t) ; b^{n}=d H^{t}\right)
$$


is the free product of cyclic group $\left\langle b ; b^{n t}=1\right\rangle$ of order $n t$ and group $H / H^{t}$ amalgamating subgroup $\left\langle b^{n}=d H^{t}\right\rangle$, and the homomorphism $\sigma_{t}$ of $B$ onto $B(t)$ extends the corresponding canonical mappings.

Now we introduce and examine the free product

$$
G_{m n}(t)=(A(t) * B(t) ; H(t))
$$

of groups $A(t)$ and $B(t)$ amalgamating subgroup $H / H^{t}$. Since the homomorphisms $\rho_{t}$ : $A \rightarrow A(t)$ and $\sigma_{t}: B \rightarrow B(t)$, clearly, coincide on the amalgamated subgroup $H$, then relative to the decomposition of group $G_{m n}=(A * B ; H)$, there exists a homomorphism $\tau_{t}: G_{m n} \rightarrow G_{m n}(t)$, extending the mappings $\rho_{t}$ and $\sigma_{t}$.

The following assertions can be easily checked.

Proposition 3.2. For any homomorphism $\varphi$ of $G_{m n}$ onto a finite group $X$, there exists an integer $t>1$ and a homomorphism $\psi$ of the group $G_{m n}(t)$ onto $X$ such that $\varphi=\tau_{t} \psi$.

Also, for any integers $t>1$ and $s>1$ such that $t$ divides $s$, there exists a homomorphism $\varphi: G_{m n}(s) \rightarrow G_{m n}(t)$ such that $\tau_{t}=\tau_{s} \varphi$. These assertions are also true for groups $A$ and $B$.

Proposition 3.3. For any element $g$ of group A (or B), not belonging to subgroup $H$, there exists an integer $t_{0}>1$ such that for any positive integer $t$ divisible by $t_{0}$, element $g \rho_{t}$ does not belong to subgroup $H \rho_{t}$ (resp., g $\sigma_{t}$ does not belong to subgroup $H \sigma_{t}$ ).

Proof. We will give the proof only for $g \in A \backslash H$. The case where $g \in B \backslash H$ is similar.

Let element $g \in A \backslash H$ and let the form $g=x_{1} x_{2} \cdots x_{r}$ be reduced relative to the decomposition of group $A$ as an amalgamated free product.

If $r=1$, then $g$ belongs to subgroup $\langle a\rangle$, that is, $g=a^{k}$ for some integer $k$ and $m$ does not divide $k$. Therefore, for any integer $t, g \rho_{t} \in\left\langle a ; a^{m t}=1\right\rangle$. Thus in group $A(t)$, element $g \rho_{t}$ cannot belong to the amalgamated subgroup (generated by element $a^{m}$ ) and consequently, it cannot belong to subgroup $H(t)=H \rho_{t}$.

Let $r>1$. Every component $x_{i}, i=1, \ldots, r$, of the reduced form of element $g$ looks like $a^{k_{i}}$, where integer $k_{i}$ is not divisible by $m$, or $c^{k_{i}} d^{l_{i}}$, where $l_{i} \neq 0$. If $x_{i}=c^{k_{i}} d^{l_{i}}\left(l_{i} \neq 0\right)$, then let $t_{0}$ be an integer which does not divide $l_{i}$ for any $i$. So, for any $t$ divisible by $t_{0}, x_{i} \rho_{t}=$ $c^{k_{i}} H^{t} d^{l_{i}} H^{t}$, where $d^{l_{i}} \notin H^{t}$. Hence, $x_{i} \rho_{t}$ will not belong to the amalgamated subgroup of group $A(t)$ (generated, we recall, by $c H^{t}$ ). Also for the components $x_{i}$ such that $x_{i}=a^{k_{i}}$, their images $x_{i} \rho_{t}$ will not belong to the amalgamated subgroup. Therefore, the form

$$
g \rho_{t}=\left(x_{1} \rho_{t}\right)\left(x_{2} \rho_{t}\right) \cdots\left(x_{r} \rho_{t}\right)
$$

of element $g \rho_{t}$ is reduced in $A(t)$ and since $r>1$, this element does not belong to its free factor $H(t)=H \rho_{t}$. The proposition is demonstrated.

From Proposition 3.3, it follows that subgroup $H$ is finitely separable in each of the groups $A$ and $B$. We then have the following proposition.

Proposition 3.4. For any element $g$ of $G_{m n}$, there exists an integer $t_{0}>1$ such that for any positive integer $t$ divisible by $t_{0}$, the length of element $g \tau_{t}$ in $G_{m n}(t)$ is equal to the length of $g$ in $G_{m n}$.

We will need finite separability of the double cosets of subgroup $H$. 
Proposition 3.5. For any elements $f$ and $g$ of $A$ (or B) such that $f$ does not belong to the double coset $\mathrm{HgH}$, there exists an integer $t_{0}>1$ such that for any positive integer $t$ divisible by $t_{0}, f \rho_{t}$ does not belong to the double coset $H(t)\left(g \rho_{t}\right) H(t)$.

Proof. We again consider only the case when elements $f$ and $g$ belong to subgroup $A$. So, suppose that $f \in A$ and does not belong to the double coset $\mathrm{HgH}$. In view of Proposition 3.2 , it is enough to prove that there exists a homomorphism $\varphi$ of $A$ onto a finite group $X$ such that element $f \varphi$ of $X$ does not belong to the double coset $(H \varphi)(g \varphi)(H \varphi)$.

To this end, let $\bar{A}=A / C$ be the quotient group of group $A$ by its (central) subgroup $C=\langle c\rangle$. The image $x C$ of an element $x \in A$ in $\bar{A}$ will be denoted by $\bar{x}$.

It is obvious that $\bar{A}$ is the (ordinary) free product of the cyclic group $X$ of order $m$ generated by $\bar{a}$, and the infinite cycle $Y$ generated by $\bar{d}$. The canonical homomorphism of $A$ onto $\bar{A}$ maps subgroup $H$ onto subgroup $Y$, and consequently the image of the double coset $H g H$ is the double coset $Y \bar{g} Y$. Since $C \leqslant H$, element $\bar{f}$ does not belong to this coset $Y \bar{g} Y$.

We can assume, without loss of generality, that any of the elements $\bar{f}$ and $\bar{g}$, if it is different from the identity, has a reduced form, where the first and the last syllables do not belong to subgroup $Y$. Every $Y$-syllable of these reduced forms can be written as $\bar{d}^{k}$, for some integer $k \neq 0$. Since the set $M$ of all such exponents $k$ is finite, we can choose an integer $t>0$ such that for any $k \in M$, the inequality $t>2|k|$ holds. We denote by $\widetilde{A}=\bar{A} /\left(\bar{d}^{t}\right)^{\bar{A}}$ the factor group of group $\bar{A}$ by the normal closure of the element $\bar{d}^{t}$ in group $\bar{A}$. The group $\tilde{A}$ is the free product of groups $X$ and $Y / Y^{t}$. Since different integers from $M$ are not congruent to one another and none are not congruent to zero modulo $t$, the reduced forms of the images $\tilde{f}$ and $\tilde{g}$ of elements $\bar{f}$ and $\bar{g}$ in $\tilde{A}$ are the same as in $\bar{A}$. In particular, $\tilde{f}$ does not belong to the double coset $Y / Y^{t} \tilde{g} Y / Y^{t}$. Since this coset consists of a finite number of elements and the group $\tilde{A}$ is residually finite, there exists a normal subgroup $\tilde{N}$ of finite index of $\tilde{A}$ such that

$$
\tilde{f} \notin\left(Y / Y^{t} \tilde{g} Y / Y^{t}\right) \cdot \tilde{N}
$$

If now $\theta$ is the product of the canonical homomorphisms of $A$ onto $\bar{A}$ and of $\bar{A}$ onto $\widetilde{A}$ and $N$ is the full preimage by $\theta$ of the subgroup $\tilde{N}$, then $N$ is a normal subgroup of finite index of $A$ and $f \notin(H g H) N$. Thus, the canonical homomorphism $\varphi$ of $A$ onto the quotient group $A / N$ has the required property and the proposition is proven.

So we quickly establish Theorem 1.2 to end with properties of group $G_{m n}$.

\section{Proof of Theorem 1.2}

We prove the following Lemma.

Lemma 4.1. Let $g \in G_{m n}$ such that $g^{-1} H g \cap H \neq 1$. Then $g$ belongs to one of the subgroups $A$ or $B$. In particular, any element of $G_{m n}$, which commutes with a nonidentity element of subgroup $H$, belongs to $A$ or $B$.

Proof. We will prove that if the length of an element $g \in G_{m n}$ is greater than 1 , then $g^{-1} \mathrm{Hg} \cap H=1$. Hence, obviously, the lemma follows. 
So let $g \in G_{m n}=(A * B ; H)$ with reduced form $g=g_{1} g_{2} \cdots g_{r}$ and let $r>1$. Assume that $g_{1} \in A$; the case when $g_{1} \in B$ is similar. Let $g^{-1} h_{1} g=h_{2}$ for some elements $h_{1}$ and $h_{2}$ of the subgroup $H$. We write this equality more precisely:

(IV)

$$
g_{r}^{-1} \cdots g_{2}^{-1} g_{1}^{-1} h_{1} g_{1} g_{2} \cdots g_{r}=h_{2} .
$$

Since the expression of the left-hand side of equality (4.1) is not reduced in $A$, we have the inclusion $g_{1}^{-1} h_{1} g_{1} \in H$. Hence, it follows by Proposition 3.1 that, $h_{1} \in\langle c\rangle$ and $g_{1}^{-1} h_{1} g_{1}=$ $h_{1}$ because $g_{1}$ does not belong to $H$ and $g_{1}^{-1} H g_{1} \cap H=\langle c\rangle$. Therefore, equality (4.1) becomes

$$
g_{r}^{-1} \cdots g_{2}^{-1} h_{1} g_{2} \cdots g_{r}=h_{2}
$$

and hence in any case $r=2$ or $r>2$, the inclusion $g_{2}^{-1} h_{1} g_{2} \in H$ takes place. Since $g_{2}$ belongs to subgroup $B$ and also does not belong to subgroup $H, h_{1} \in\langle d\rangle$ because $g_{2}^{-1} \mathrm{Hg}_{2} \cap$ $H=\langle d\rangle$ (see Proposition 3.1). Thus, $h_{1} \in\langle c\rangle \cap\langle d\rangle=1$. Consequently, $h_{1}=1$ as required.

Proof of Theorem 1.2. Let $U$ be an abelian subgroup of group $G_{m n}$ and let $U$ contain a cyclically reduced element $u=u_{1} u_{2} \cdots u_{r}$ of length greater than 1 . It is not difficult to see that any other nonidentity element $v$ of $U$ is cyclically reduced of length greater than 1 .

Indeed, suppose that $v=v_{1} v_{2} \cdots v_{s}$ is another nonidentity element of $U$. So $u v=v u$. Then certainly $v \notin H$, since $U \cap H=1$. If $s=1$, then $v$ belongs to one of the subgroups $A$ or $B$ together with one of the elements $u_{1}$ or $u_{r}$. Let for example $v$ and $u_{1}$ belong to the same subgroup $A$ or $B$ and let $u_{r}$ and $v$ belong to different subgroups $A$ and $B$. From $u v=v u$, we have the equality

$$
u_{1} u_{2} \cdots u_{r} v=v u_{1} u_{2} \cdots u_{r}
$$

from which the left-hand side has length $r+1$, while the right-hand side has length $r-1$ or $r$, depending on whether element $v u_{1}$ belongs to $H$ or not. Hence, this inequality of lengths is a contradiction which proves that $s>1$. Now suppose that $v$ is not cyclically reduced. This means that elements $v_{1}$ and $v_{s}$ belong to the same subgroup $A$ or $B$ and this subgroup contains one of the elements $u_{1}$ and $u_{r}$; let it be $u_{1}$. Then from the equality $u v=v u$, that is,

$$
u_{1} u_{2} \cdots u_{r} v_{1} v_{2} \cdots v_{s}=v_{1} v_{2} \cdots v_{s} u_{1} u_{2} \cdots u_{r}
$$

the left-hand side has length $r+s$, while the right-hand side has length $r+s-1$; this is again impossible. So, element $v$ is cyclically reduced, and consequently any nonidentity element from $U$ is cyclically reduced and has length greater than 1 .

Let $u$ be now a nonidentity element of minimum length from subgroup $U$ and let $u=u_{1} u_{2} \cdots u_{r}$ be reduced. We claim that subgroup $U$ is generated by $u$. Namely, for any nonidentity element $v \in U$, we will prove by induction on $l(v)$ that $v$ is equal to some power of $u$. 
Let $v=v_{1} v_{2} \cdots v_{s}$ be a reduced form of such an element $v$. We recall that by the selection of the element $u$, we have the inequality $s \geqslant r$. If we replace, when necessary, $v$ by $v^{-1}$, we can, without loss of generality, consider that elements $u_{1}$ and $v_{1}$ belong to the same subgroup $A$ or $B$. Then $u_{r}$ and $v_{1}$, as well as elements $u_{1}$ and $v_{s}$, also belong to different subgroups, and consequently, the forms of both parts of the equality

$$
u_{1} u_{2} \cdots u_{r} v_{1} v_{2} \cdots v_{s}=v_{1} v_{2} \cdots v_{s} u_{1} u_{2} \cdots u_{r}
$$

are reduced. By the equalities in (2.2), there exist elements $h_{0}, h_{1}, \ldots, h_{r+s}$ of subgroup $H$ such that

$$
u_{i}=h_{i-1}^{-1} v_{i} h_{i} \quad(1 \leq i \leq r+s) \quad h_{0}=h_{r+s}=1 .
$$

Hence, $u=v_{1} v_{2} \cdots v_{r} h_{r}$, and consequently, if $s=r$, we have $u=v h_{r}$. But then, $h_{r}=$ $v^{-1} u \in U \cap H$. Hence $h_{r}=1$ and $v=u$. So, if $s=r$, our statement is satisfied.

If $s>r$, we have $v=u v^{\prime}$, where $v^{\prime}=h_{r}^{-1} v_{r+1} \cdots v_{s}$ and the length of element $v^{\prime} \in U$ is less than $s$. Hence, by induction, $v^{\prime}=u^{k}$ for some integer $k$. Therefore, $v=u^{k+1}$, and hence the proof is complete.

We now proceed to the proof of Theorem 1.1.

\section{Proof of Theorem 1.1}

Before we go through this proof, we note that for any $t>1$, each of the groups $A(t)$ and $B(t)$ is the amalgamated free product of two finite groups. Hence, $A(t)$ and $B(t)$ are freeby-finite [6], and therefore they are $\pi_{c}[7]$.

We also see that $A$ and $B$ are free products of finitely generated abelian groups amalgamating cyclic subgroups. Thus by [8] or [10], groups $A$ and $B$ are $\pi_{c}$.

So, to prove Theorem 1.1, it is enough to show that for any two elements $f$ and $g$ of $G_{m n}$, where $f \notin\langle g\rangle$, there exists an integer $t>1$ such that $f \tau_{t} \notin\langle g\rangle \tau_{t}$. Then it will be possible to find a homomorphism $\psi$ of $G_{m n}(t)$ onto a finite group $X$ such that $\left(f \tau_{t}\right) \psi \notin\langle g\rangle\left(\tau_{t}\right) \psi$, and consequently the product $\tau_{t} \psi$ will be the required homomorphism of group $G_{m n}$ onto a finite group.

Now let $f$ and $g$ be a pair of elements of $G_{m n}$ such that $f \notin\langle g\rangle$. We can assume that $g$ is cyclically reduced. Group $G_{m n}$ is residually finite as was mentioned. So we consider $g \neq 1$ and clearly $f \neq 1$.

If $f \notin\langle g\rangle$, then by Proposition 2.2, we have the following cases.

(1) Elements $f$ and $g$ belong to the same factor $A$ or $B$ and $f \neq g^{k}$ for all integers $k$.

(2) Elements $f$ and $g$ belong to different factors $A$ and $B$ and $f \neq g^{k}$ for all integers $k$.

(3) $l(g)=1$ and $l(f)>1$.

(4) $l(f)=1$ and $l(g)>1$.

(5) $l(f)>1, l(g)>1$ and $l(g)$ does not divide $l(f)$.

(6) $l(f)>1, l(g)>1$ and $l(g)$ divides $l(f)$, but (2.4) is not satisfied.

We prove separately the above cases.

Case (1). Assume first that both elements lie in one of the subgroups $A$ or $B$; let it be $A$ for instance. Since $f \notin\langle g\rangle$ and since $A$ is $\pi_{c}$, there exists a homomorphism $\varphi$ of $A$ onto 
some finite group $X$ such that $f \varphi \notin\langle g\rangle \varphi$. By Proposition 3.2, there exist an integer $t>1$ and a homomorphism $\psi$ of $A(t)$ onto $X$ such that $\varphi=\rho_{t} \psi$. This means that $f \rho_{t} \notin\langle g\rangle \rho_{t}$ in $A(t)$. Thus, by Proposition 2.2, for the given integer $t$, in group $G_{m n}(t)$, elements $f \tau_{t}$ and $g \tau_{t}$ belong to the free factor $A(t)$ and $f \tau_{t} \notin\langle g\rangle \tau_{t}$.

Case (2). Let elements $f$ and $g$ now lie in different subgroups $A$ and $B$; for example let $f$ belong to $A$, let $g$ belong to $B$, and each of these elements does not belong to the subgroup $H$. By Proposition 3.3, there exists an integer $t_{1}>1$ such that for any positive integer $t$ divisible by $t_{1}, f \rho_{t}$ does not belong to $H \rho_{t}$, and there exists an integer $t_{2}>1$ such that for any positive integer $t$ divisible by $t_{2}, g \sigma_{t}$ does not belong to $H \sigma_{t}$. Thus, at $t=t_{1} t_{2}$, elements $f \tau_{t}$ and $g \tau_{t}$ belong to different free factors $A(t)$ and $B(t)$ of $G_{m n}(t)$ and also do not belong to the amalgamated subgroup $H(t)$ relative to the decomposition of $G_{m n}(t)$. Hence, $f \tau_{t} \notin\langle g\rangle \tau_{t}$ since otherwise, by Proposition 2.2, this would imply that elements $f \tau_{t}$ and $g \tau_{t}$ belong to the amalgamated subgroup $H(t)$.

Case (3). $l(g)=1$ and $l(f)>1$. thus, $f \notin\langle g\rangle$ by Proposition 2.2.

From Proposition 3.4, it follows that there exists an integer $t_{1}>1$ such that for any positive integer $t$ divisible by $t_{1}$, the length of $f \tau_{t}$ in $G_{m n}(t)$ is equal to the length of $f$ in $G_{m n}$; that is, $l\left(f \tau_{t}\right)>1$. Similarly, there exists an integer $t_{2}>1$ such that for any positive integer $t$ divisible by $t_{2}, l\left(g \tau_{t}\right)=1$ in $G_{m n}(t)$. Thus, at $t=t_{1} t_{2}, l\left(f \tau_{t}\right)>1$ and $l\left(g \tau_{t}\right)=1$ in $G_{m n}(t)$. Hence, from Proposition 2.2, $f \tau_{t} \notin\langle g\rangle \tau_{t}$ and the required integer $t$ is found.

Case (4). $l(g)>1$ and $l(f)=1$. The proof of this case is similar to the proof of case (3) above.

Case (5). $l(f)>1, l(g)>1$, and $l(g)$ does not divide $l(f)$.

From Proposition 3.4, there exists an integer $t_{1}>1$ such that for any positive integer $t$ divisible by $t_{1}, l\left(f \tau_{t}\right)=l(f)$. Similarly, there exists an integer $t_{2}>1$ such that for any positive integer $t$ divisible by $t_{2}, l\left(g \tau_{t}\right)=l(g)$. Thus at $t=t_{1} t_{2}$, elements $f \tau_{t}$ and $g \tau_{t}$ of $G_{m n}(t)$ are such that $l\left(f \tau_{t}\right)>1, l\left(g \tau_{t}\right)>1$, and $l\left(g \tau_{t}\right)$ does not divide $l\left(f \tau_{t}\right)$. Hence, from Proposition 2.2, $f \tau_{t}$ cannot belong to the subgroup $\langle g\rangle \tau_{t}$.

Case (6). $l(f)>1, l(g)>1$, and $l(g)$ divides $l(f)$ but (2.4) is not satisfied.

Let $f=x_{1} x_{2} \cdots x_{r}$ be reduced and let $g=y_{1} y_{2} \cdots y_{s}$ be cyclically reduced. By Proposition 2.2, if $f=g^{k}$ for some integer $k \neq 0$, then $r=k s$ and there exist elements $h_{0}, h_{1}, \ldots, h_{k s}$ of $H$ such that property (2.4) is satisfied. Thus elements $x_{i}$ and $y_{i}(1 \leq i \leq k s)$ belong to the same free factor and define the same double coset of $H$ and $y_{j}=y_{j+l s}$ for $1 \leq j \leq s$ and $0 \leq l \leq k-1$.

So, if $f \notin\langle g\rangle$, three subcases arise.

Subcase (i). Suppose that, for some integer $i=1, \ldots, k s$, elements $y_{i}$ and $x_{i}$ lie in different free factors $A$ and $B$ of group $G_{m n}$ (and, certainly, are not in the subgroup $H$ ). Then as in case (2) above, there exists an integer $t_{i}>1$ such that for any positive integer $t$ divisible by $t_{i}$, elements $x_{i} \tau_{t}$ and $y_{i} \tau_{t}$ belong to the different free factors $A(t)$ and $B(t)$, respectively, and do not belong to the amalgamated subgroup $H(t)$ of $G_{m n}(t)$. Also by Proposition 3.4, there exist integers $t^{\prime}>1$ and $t^{\prime \prime}>1$ such that for any positive integer $t$ divisible by $t^{\prime}$, the length of $f \tau_{t}$ in $G_{m n}(t)$ is equal to $r$ and for any positive integer $t$ divisible by $t^{\prime \prime}$, the 
length of $g \tau_{t}$ in $G_{m n}(t)$ is equal to $s$. Thus, at $t=t_{i} t^{\prime} t^{\prime \prime}$, elements $f \tau_{t}$ and $g \tau_{t}$ have reduced forms

$$
\left(x_{1} \tau_{t}\right)\left(x_{2} \tau_{t}\right) \cdots\left(x_{r} \tau_{t}\right), \quad\left(y_{1} \tau_{t}\right)\left(y_{2} \tau_{t}\right) \cdots\left(y_{s} \tau_{t}\right)
$$

respectively, in $G_{m n}(t)$ and $x_{i} \tau_{t}$ and $y_{i} \tau_{t}$ belong to different free factors $A(t)$ and $B(t)$, respectively, and do not belong to the amalgamated subgroup $H(t)$. Hence, by Proposition 2.2, $f \tau_{t}$ cannot belong to subgroup $\langle g\rangle \tau_{t}$ in group $G_{m n}(t)$.

Subcase (ii). Let now, for all $i=1, \ldots, k s$, elements $y_{i}$ and $x_{i}$ belong to the same subgroup $A$ or $B$ and for some $j(j=1, \ldots, k s)$, element $x_{j}$ does not belong to the double coset $H y_{j} H$. Suppose that $x_{j}$ and $y_{j}$ both belong to $A$. By Proposition 3.5, there exists an integer $t_{j}>1$ such that for any positive integer $t$ divisible by $t_{j}$, element $x_{j} \rho_{t}$ is not in the double coset $H / H^{t}\left(y_{j} \rho_{t}\right) H / H^{t}$. Also by Proposition 3.4, there exist integers $t^{\prime}>1$ and $t^{\prime \prime}>1$ such that, for any positive integer $t$ divisible by $t^{\prime}$, the length of $f \tau_{t}$ in $G_{m n}(t)$ is equal to $r$ and for any positive integer $t$ divisible by $t^{\prime \prime}$, the length of $g \tau_{t}$ in $G_{m n}(t)$ is equal to $s$. Since $r=k s$, at $t=t_{j} t^{\prime} t^{\prime \prime}$, elements $f \tau_{t}$ and $g \tau_{t}$ have reduced forms

$$
\left(x_{1} \tau_{t}\right)\left(x_{2} \tau_{t}\right) \cdots\left(x_{r} \tau_{t}\right), \quad\left(y_{1} \tau_{t}\right)\left(y_{2} \tau_{t}\right) \cdots\left(y_{r} \tau_{t}\right)
$$

respectively, in $G_{m n}(t)$ and $x_{j} \tau_{t}$ does not lie in the double coset $H / H^{t}\left(y_{j} \tau_{t}\right) H / H^{t}$. Hence by Proposition 2.2, $f \tau_{t}$ cannot belong to subgroup $\langle g\rangle \tau_{t}$ in $G_{m n}(t)$, since (2.4) is not satisfied. Thus integer $t$ is found.

Subcase (iii). We suppose now that for some $i, y_{i} \neq y_{i+l s}, 1 \leq i \leq s$, and $1 \leq l \leq k-1$.

(a) If these elements $y_{i}$ and $y_{i+l s}$ belong to the same subgroup $A$ or $B$, then as in case (1) above, since groups $A$ and $B$ are residually finite, there exists a homomorphism $\varphi$ of group $A$ (or $B$ ) onto some finite group $X$ such that $y_{i} \varphi \neq y_{i+l s} \varphi$. But since by Proposition 3.2 any homomorphism of group $A$ (group $B$ ) onto a finite group factors through some group $A(t)$ (resp., $B(t)$ ), for this $t$, there exists homomorphism $\psi$ of group $A(t)$ (or $B(t)$ ) onto $X$ such that $\varphi=\rho_{t} \psi$. Then, in the free factor and thus in $G_{m n}(t), y_{i} \rho_{t} \neq y_{i+l s} \rho_{t}$, and so condition (2.4) for the inclusion of element $f \tau_{t}$ in subgroup $\langle g\rangle \tau_{t}$ will not be satisfied.

(b) If these elements $y_{i}$ and $y_{i+l s}$ belong to different subgroups $A$ and $B$, then similar to case (2), for some value of $t$, elements $y_{i} \tau_{t}$ and $y_{i+l s} \tau_{t}$ belong to different free factors $A(t)$ and $B(t)$ of group $G_{m n}(t)$ and also do not belong to the amalgamated subgroup $H(t)$. Thus, in group $G_{m n}(t), y_{i} \tau_{t} \neq y_{i+l s} \tau_{t}$ and condition (2.4) of Proposition 2.2 will not be satisfied. So, element $f \tau_{t}$ cannot be in subgroup $\langle g\rangle \tau_{t}$. Hence we have the proof of Theorem 1.1.

\section{Acknowledgments}

The author gratefully acknowledges the financial support from the International Mathematical Union (IMU) CDE Exchange Programme and The Abdus Salam International Centre for Theoretical Physics (ICTP) for the visit to the ICTP in summer 2004. 


\section{Abelian subgroup separability of some one-relator groups}

\section{References}

[1] M. Hall Jr., Coset representations in free groups, Trans. Amer. Math. Soc. 67 (1949), 421-432.

[2] E. D. Loginova, Residual finitness of free products with commuting subgroups, Siberian Math. J. 40 (1999), no. 2, 341-350, Translation from Sib. Mat. Zh. 40 (2) (1999), 395-407.

[3] W. Magnus, A. Karrass, and D. Solitar, Combinatorial Group Theory: Presentations of Groups in Terms of Generators and Relations, Interscience Publishers [John Wiley \& Sons], New York, 1966.

[4] A. I. Malcev, On isomorphic matrix representations of infinite groups, Rec. Math. [Mat. Sbornik] N.S. 8 (50) (1940), 405-422.

[5] _ On homomorphisms onto finite groups, Amer. Math. Soc. Transl. Ser. 2119 (1983), 67-79, Translation from Ivanov. Gos. Ped. Inst. Ucen. Zap. 18 (1958) 49-60.

[6] B. H. Neumann, An essay on free products of groups with amalgamations, Philos. Trans. Roy. Soc. London Ser. A 246 (1954), 503-554.

[7] P. F. Stebe, Residual solvability of an equation in nilpotent groups, Proc. Amer. Math. Soc. 54 (1976), 57-58.

[8] C. Y. Tang, On the subgroup separability of generalized free products of nilpotent groups, Proc. Amer. Math. Soc. 113 (1991), no. 2, 313-318.

[9] D. Tieudjo and D. I. Moldavanski, Endomorphisms of the group $G_{m n}=\left\langle a, b ;\left[a^{m}, b^{n}\right]=\right.$ $1\rangle(m, n)>1$, Afrika Mat. (3) 9 (1998), 11-18.

[10] P. C. Wong and H. L. Koay, Generalized free products of $\pi_{c}$ groups, Rocky Mountain J. Math. 22 (1992), no. 4, 1589-1593.

[11] P. C. Wong and C. K. Tang, Cyclic subgroup separability of certain HNN extensions of finitely generated abelian groups, Rocky Mountain J. Math. 29 (1999), no. 1, 347-356.

D. Tieudjo: Department of Mathematics and Computer Science, University of Ngaoundere, P.O. Box 454, Ngaoundere, Cameroon

E-mail address: tieudjo@yahoo.com 


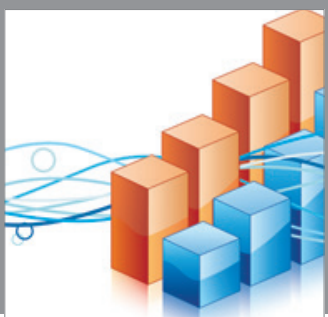

Advances in

Operations Research

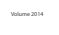

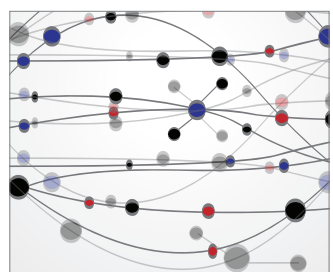

\section{The Scientific} World Journal
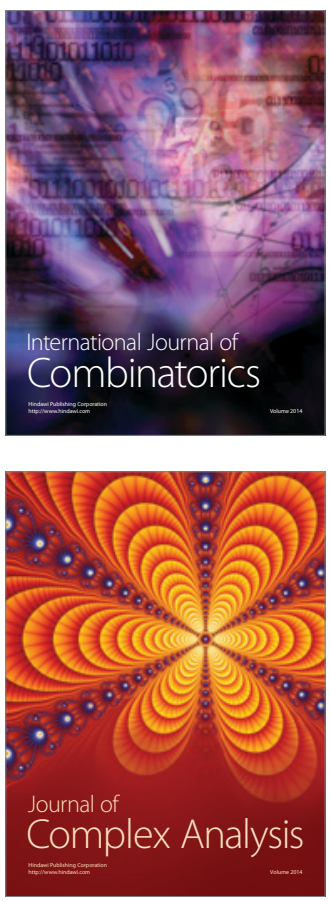

International Journal of

Mathematics and

Mathematical

Sciences
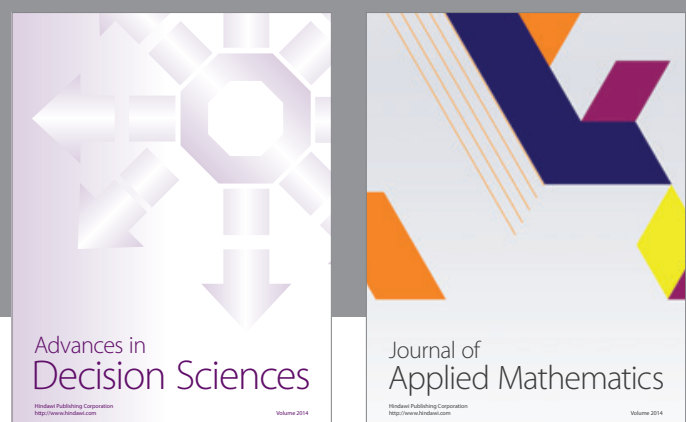

Journal of

Applied Mathematics
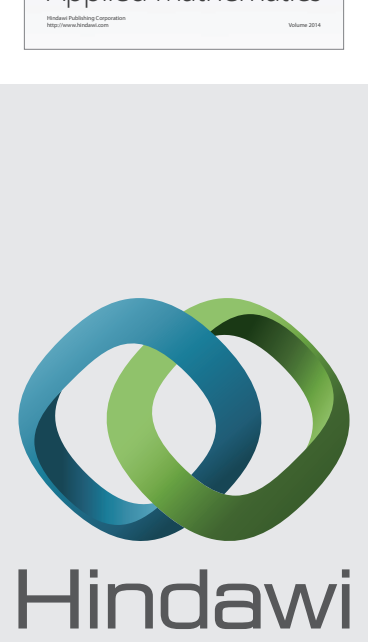

Submit your manuscripts at http://www.hindawi.com
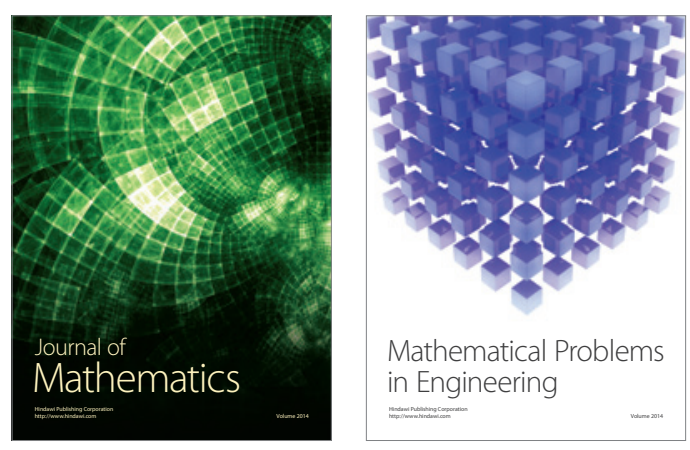

Mathematical Problems in Engineering
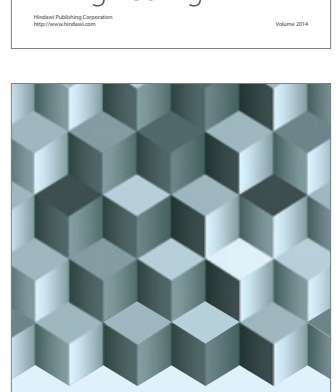

Journal of

Function Spaces
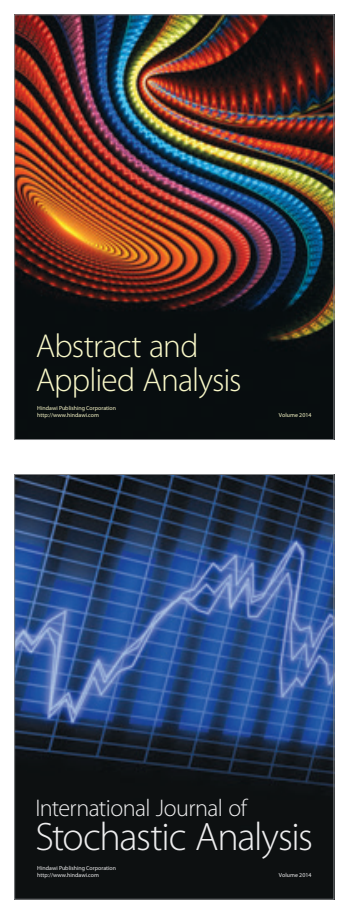

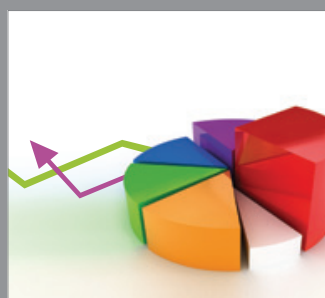

ournal of

Probability and Statistics

Promensencen
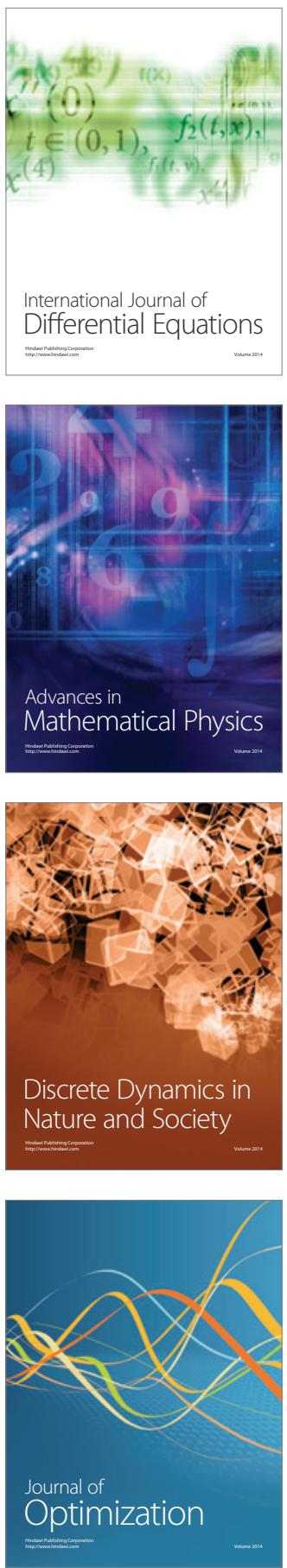